\title{
Qualitätsmanagementsysteme - eine Analyse von Diffusionsprozessen in verschiedene organisationale Felder der Weiterbildung
}

\author{
Martin Reuter • Andreas Martin · Stefan Koscheck
}

Eingegangen: 28. Juni 2019 / Angenommen: 13. September 2019 / Online publiziert: 18. Oktober 2019 (C) Der/die Autor(en) 2019

Zusammenfassung Qualitätsmanagementsysteme sind mittlerweile fester Bestandteil der Erwachsenenbildung. Die Etablierung dieser ursprünglich aus der Industrie stammenden Managementinstrumente kann als Diffusionsprozess beschrieben werden. Weitgehend ungeklärt ist bisher jedoch, inwiefern sich Organisationen dabei an ihrer organisationalen Feldzugehörigkeit orientierten, und welche Bedeutung bildungspolitische Interventionen in Form von gesetzlichen Vorgaben für die Gestaltung dieser Diffusionsprozesse besaßen. Im Sinne der Pluralität der Weiterbildung werden verschiedene Kontexte voneinander getrennt betrachtet. Theoretisch wird an den Neo-Institutionalismus angeschlossen. Auf Basis der Daten der wbmonitor-Umfrage 2017 wird eine Ereignisdatenanalyse durchgeführt. Diese ermöglicht es zu untersuchen, inwiefern das organisationale Feld und gesetzliche Vorgaben einen Einfluss auf die Implementierungswahrscheinlichkeit von Qualitätsmanagementsystemen besitzen. Im Ergebnis zeigen sich trotz eines ähnlichen Gesamtverlaufs bei der Implementierung auch Unterschiede zwischen den organisationalen Feldern (1 „VHS“, 2 ,gemeinnützig/öffentlich“, 3 „Gemeinschaften“ und 4 ,,kommerziell privat"). Außerdem können deutliche Effekte der gesetzlichen Vorgaben belegt werden, wobei auch diese unterschiedlich in den Feldern wirken.

\footnotetext{
M. Reuter, M.A. $(\bowtie)$

Justus-Liebig-Universität Gießen, Gießen, Deutschland

E-Mail: martin.reuter@erziehung.uni-giessen.de

Dr. A. Martin

Deutsches Institut für Erwachsenenbildung, Leibniz-Zentrum für lebenslanges Lernen e. V., Bonn, Deutschland

E-Mail: martin@die-bonn.de

S. Koscheck

Bundesinstitut für Berufsbildung, Bonn, Deutschland

E-Mail: koscheck@bibb.de
} 
Schlüsselwörter Qualitätsmanagement · Steuerung $\cdot$ Erwachsenenbildung $\cdot$ NeoInstitutionalismus · Isomorphien $\cdot$ AZAV $\cdot$ Ereignisdatenanalyse

\title{
Quality management systems-analysing processes of diffusion in different organizational fields of continuing education
}

\begin{abstract}
Quality management systems have become an integral part of adult education. The process of establishing these management tools, which originally stem from the industrial sector, can be described as process of diffusion. Until now, the extent of organizations orientations towards the expectations of their organizational field affiliation and the significance of educational policy interventions, such as legal requirements for the design of these diffusion processes, has largely remained unknown. With regard to the plurality of continuing education, different contexts are considered. New-institutionalism will be used as main approach. Based on the data of the wbmonitor-survey 2017, an event history analysis is methodically carried out. This allows examining to what extent the organizational field and legal requirements have an influence on the probability of implementing quality management systems. Despite a similar development of the implementation process, differences between the organizational fields (1 "VHS", 2 "non-profit/public domain", 3 "commuities/ associations" and 4 "private sector") are showing. Furthermore, clear effects of legal requirements are visible, although these requirements have different effects on the respective fields as well.
\end{abstract}

Keywords Quality management - Governance · Adult education · New institutionalism $\cdot$ Isomorphism $\cdot \mathrm{AZAV} \cdot$ Event history analysis

\section{Qualitätsmanagementsysteme in der Weiterbildung}

Im Kontext der Steuerung des Weiterbildungsbereichs spielt der Qualitätsdiskurs seit nunmehr über 20 Jahren eine prominente Rolle (vgl. Hartz 2018; Käpplinger 2017) und kann ,[n] ach verbreiteter Auffassung [... als] eine eher neo-liberale Modernisierungsstrategie" (Schrader 2011, S. 26) gedeutet und analysiert werden. $\mathrm{Zu}$ Beginn der Diskussion machte sich Gieseke für eine aktive Beteiligung an der Debatte im Sinne weiter zu verfolgender Professionalisierung stark (vgl. Gieseke 1997). Der Professionalisierungsdiskurs wurde bis dahin primär im Bereich der öffentlich-rechtlich reglementierten Weiterbildung geführt (vgl. ebd.; Schrader 2011, S. 72). Im Unterschied dazu wird der Qualitätsdiskurs in der Weiterbildung, obgleich dessen Ursprung im industriellen Sektor liegt, bereichsübergreifend geführt (vgl. ebd.). Der spezifische Fokus liegt dabei auf der Organisationsebene (vgl. Ehses 2016, S. 151). Dies kann als Suche nach einem ,für die Gesamtheit der Bildungsorganisationen geltenden Organisations- und Handlungssystem“ (Meisel 2002, S. 9f.) gedeutet werden.

Neue, zentrale Form der Steuerung des öffentlichen Bereichs ist das „New Public Management" (NPM), das sich besonders im Bereich der öffentlichen Verwaltung etabliert hat (vgl. Dewe 2005; Vogel 2007). Vor allem über diesen Weg wurden 
Qualitätsmanagementkonzepte in die Weiterbildung getragen. Zentrales Element des NPM ist die ergebnisorientierte Steuerung, die stark auf den Druck von Märkten bzw. Quasi-Märkten setzt. Diese Bedeutungszunahme betriebswirtschaftlicher Elemente hat auch in der Weiterbildung zu kritischen Auseinandersetzungen mit Qualitätsmanagement (QM) geführt, in deren Rahmen diese beispielsweise machttheoretisch als ,neue Beichtpraxis“ (Forneck und Wrana 2005, S. 174) oder mikropolitisch als „Fluch oder Segen“ (Käpplinger 2017) analysiert wurden. Eine Analyse zum Verhältnis von NPM und der Professionalisierung des Bildungsbereichs liegt von Schimank (2015) vor. Der bildungspolitische Ansatz, Qualitätssicherung in der Weiterbildung einzuführen, lag (ab Ende der 1990er Jahre) insbesondere darin, die Adaption aus der Industrie stammender Qualitätsmanagementsysteme (QMS) mit zentralem Fokus auf der internationalen ISO Normreihe $9000 \mathrm{ff}$. zu fördern. Dabei war die Annahme leitend, dass QMS, die in der Produktion von Gütern eine zuverlässige und gleichbleibende, vorab definierte Qualität gewährleisten sollen (vgl. Egetenmeyer und Käpplinger 2011, S. 25 f.), auf pädagogische Prozesse übertragbar sind - ungeachtet des dortigen Technologiedefizits (vgl. Luhmann und Schorr 1982). ${ }^{1}$ Im Fokus standen damit sowohl die Verbesserung organisationaler als auch pädagogischer Prozesse und Ergebnisse.

Ein wichtiger Aspekt der Forcierung von QMS in der Weiterbildung ist zudem in der Legitimierung staatlicher Förderentscheidungen zu sehen. So wurde auf Bundesebene ein QMS Voraussetzung, um beispielsweise im Bereich der Arbeitsförderungsmaßnahmen (SGB III/II) tätig werden zu dürfen. Rechtlich fixiert wurde dies in der „Anerkennungs- und Zulassungsverordnung Weiterbildung 2004“ (AZWV), welche 2012 in die „Akkreditierungs- und Zulassungsverordnung Arbeitsförderung“ (AZAV) umbenannt wurde. Auf Länderebene werden QMS im Kontext der Anerkennung als förderfähige Einrichtung in den „Weiterbildungsgesetzen“(WBG) teilweise festgeschrieben.

Die Etablierung von QMS in der Weiterbildung lässt sich als Diffusionsprozess beschreiben. Hartz (vgl. 2009, 2011) untersuchte diesbezüglich das QMS „Lernerorientierte Qualitätstestierung“ (LQW). In der gesamten Weiterbildung war bis ca. Mitte der 1990er Jahre die Implementation eines QMS vergleichsweise selten. Dann, deutlich zunehmend, erreichte die Häufigkeit der Implementierung pro Jahr 2005 gemäß der deskriptiven Auswertung der Ergebnisse des wbmonitors - den bisherigen Höhepunkt und fiel danach insgesamt wieder ab (vgl. Ambos et al. 2018, S. 22 ff.). 2017 verfügten $80 \%$ der Weiterbildungsorganisationen über ein QMS (ebd., S. 12), und weitere 5\% planten die Einführung ${ }^{2} ; 52 \%$ aller Organisationen waren extern zertifiziert (vgl. ebd., S. 12). Insgesamt zeigt sich gegenwärtig eine hohe Verbreitung im gesamten Anbieterspektrum der Weiterbildung. Wurden zu Beginn branchenunspezifische Modelle adaptiert, differenzierten sich branchenspezifische Modelle Anfang der 2000er Jahre deutlich aus, sodass mittlerweile eine Vielzahl an QMS unterschiedlicher Ausrichtung existieren. ${ }^{3}$ Die DIN ISO $9000 \mathrm{ff}-$

\footnotetext{
1 Vgl. dazu kritisch Käpplinger und Reuter (2017).

2 Quelle: eigene Berechnung auf Basis der wbmonitor-Umfrage 2017.

${ }^{3}$ Für eine Übersicht bzw. einen Vergleich zentraler QMS vgl. Käpplinger und Reuter (2017, S. 12f.) oder Ambos et al. (2018, S. $17 \mathrm{ff}$.).
} 
Normenreihe - eines der ersten QM-Modelle überhaupt - wird mit Abstand am häufigsten eingesetzt (35\% aller Organisationen; vgl. ebd., S. 14f.).

Nicht nur in der Weiterbildung insgesamt, sondern auch in deren verschiedenen Teilsegmenten besteht bei den Organisationen ein hoher Verbreitungsgrad von QMS. So finden sich in den vier organisationalen Feldern - ,VHS”, ,gemeinnützig/öffentlich“, „Gemeinschaften“ und „,kommerziell privat“ - die Reuter, Koscheck und Martin (vgl. 2019) mittels einer empirischen, mehrdimensionalen Systematisierung identifizierten, jeweils ein Abdeckungsgrad an QMS von $75 \%$ bis $82 \%$. Unterschiede zeigen sich allerdings hinsichtlich der Art der genutzten QM-Modelle.

Für die Analyse von Diffusionsprozessen eignet sich die Theorie des organisationssoziologischen Neo-Institutionalismus, welche das Entstehen von organisationalen Strukturen und Verfahren durch Prozesse der Isomorphie (Gleichgestaltigkeit) erklärt. Zudem erlaubt dieser Ansatz mit der Unterscheidung organisationaler Felder eine Betrachtung verschiedener Bereiche der Weiterbildung. Daher gilt es zunächst, auf Theoriegrundlagen sowie zentrale Begriffe einzugehen. Daran anschließend werden die Forschungsfrage konkretisiert und Hypothesen abgeleitet. Sodann wird das methodische Vorgehen erläutert, bevor die erwähnten Diffusionsprozesse genauer beschrieben werden. Der Artikel schließt mit der Diskussion der Ergebnisse.

\section{Theoretische Aspekte im Anschluss an den Neo-Institutionalismus}

Der organisationssoziologische Neo-Institutionalismus nimmt Organisationen an der Schnittstelle zur Gesellschaft in den Blick. Organisationen und ihre Strukturen kommen so in einem Bedingungsfeld in den Fokus. Maßgeblich für dieses Feld ist, dass es aus Institutionen (Normen, Erwartungen, Leitbilder) besteht (vgl. Herbrechter und Schemmann 2010, S. 128). In diesem Feld sind Organisationen ,durch gesellschaftliche Erwartungen im Allgemeinen und durch staatlich-politische Regulierungen im Besonderen beeinflusst“" (Hasse und Krücken 2005, S. 55). Die Analyse der Diffusion solcher organisationsexterner gesellschaftlicher Erwartungen in Organisationen ist Gegenstand neo-institutionalistischer Forschung (vgl. ebd.).

Als theoretische Klassiker gelten besonders die Arbeiten von Meyer und Rowan (1977, deutsche Übersetzung 2009) und DiMaggio und Powell (1983, deutsche Übersetzung 2009). Gingen vorher bürokratietheoretische Annahmen davon aus, dass das Streben nach Effizienz die Strukturen einer Organisation forme und diese legitimiere (vgl. Weber 1972), brechen Meyer und Rowan damit und betonen, dass es primär um Legitimitätserhalt gehe und der Aspekt der Effizienz dafür nachrangig sei. Legitimität erhielten Organisationen dadurch, dass sie an als rational angesehene Umwelterwartungen anschließen (vgl. Meyer und Rowan 1977, 2009). Dabei ist der Begriff „Rationalitätsmythen“ zentral.

Die in die Gesellschaft eingebetteten Regeln haben insofern eine rationale Dimension, als dass soziale Ziele sowie Mittel zur Verfolgung solcher Ziele festgelegt werden. Die mythische Dimension folgt aus der Tatsache, dass die Wirksamkeit solcher Mittel nicht bewiesen ist, sondern lediglich an deren Erfolg geglaubt wird (Schemmann 2018, S. 189). 
Folge dessen ist eine Angleichung von Organisationen an ihre Umwelt. DiMaggio und Powell (vgl. 1983) schließen an die Fokussierung der Legitimation an, wobei sie den Aspekt der Angleichung konkretisieren und die Umwelt als ,organisationales Feld" konzipieren. Als solches bezeichnen sie

jene Organisationen, die als Aggregat einen erkennbaren Bereich des institutionellen Lebens konstituieren: Zentrale Lieferanten, Konsumenten von Ressourcen und Produkten, Regulierungsbehörden sowie andere Organisationen, die ähnliche Dienstleistungen oder Produkte erstellen (DiMaggio und Powell 2009, S. 59).

Damit nehmen sie auch Angleichungsprozesse zwischen Organisationen in den Blick. Hervorzuheben ist, dass das organisationale Feld als zentrale Analyseeinheit im Neo-Institutionalismus gesehen wird (vgl. Senge 2005) und nur empirisch bestimmt werden kann (vgl. DiMaggio und Powell 2009, S. 64). Damit ist ein Verständnis des organisationalen Feldes gegeben, das mit einem mittleren Komplexitätsgrad eine gewisse Differenziertheit bietet, ohne für die empirische Operationalisierung überkomplex zu sein. ${ }^{4}$

Strukturangleichungen zwischen Organisationen werden mit dem „Konzept der Isomorphie“" gefasst. ${ }^{5}$ Diese Isomorphie wird in die drei Mechanismen Zwang (Organisationen werden beispielsweise durch Gesetze zu einer bestimmten Handlung gezwungen), Mimese (Organisationen beobachten andere erfolgreiche ihres Feldes und ahmen diese nach) und Norm (Anpassung formaler Strukturen an professionelle Standards) differenziert. Gleichwohl ist die Praxis dadurch gekennzeichnet, dass die drei Mechanismen nicht in Einzelform auftreten, sondern in einer sich wechselseitig beeinflussenden Beziehung zueinanderstehen (vgl. DiMaggio und Powell 1983, S. 150; Hartz 2009, S. 138; auch Hasse und Krücken 2005, S. 27; Krücken 2004;).

\section{Forschungsfrage und Hypothesen}

An die theoretischen Annahmen anschließend, dass sich die Diffusion von QMS in die Weiterbildung gemäß des Neo-Institutionalismus als isomorpher Prozess beschreiben lässt (vgl. Hartz 2009), und die Umwelt der Organisationen, die mit dem Konzept des organisationalen Feldes (DiMaggio und Powell 1983, 2009) dahingehend spezifiziert werden kann, dass dieses einen besonderen Orientierungsrahmen für die zugehörigen Organisationen darstellt, wird im Folgenden die Wirkung des organisationalen Feldes auf die Organisationsentscheidungen hinsichtlich der Einführung von QMS untersucht. Dabei ist davon auszugehen, dass es sich bei den hier wirksamen Mechanismen zum einen um Norm und Mimese und zum anderen um Zwang handelt, wobei es bei den beiden erstgenannten schwierig erscheint, diese in der Analyse unterscheiden zu können. Insgesamt ist davon auszugehen, dass mehre-

\footnotetext{
${ }^{4}$ Zur Übersicht von Weiterführungen des Konzepts vgl. Becker-Ritterspach und Becker-Ritterspach (2006, S. 118 ff.).

${ }^{5}$ Zur kritischen Diskussion der Reichweite der Isomorphie vgl. Becker-Ritterspach und Becker-Ritterspach (2006).
} 
re oder alle Mechanismen auf die Strukturen von Organisationen wirken und zudem miteinander in Wechselwirkung stehen.

Unter der theoretischen Annahme, dass Organisationen an Umwelterwartungen respektive deren Rationalitätsmythen anschließen, wird davon ausgegangen, dass die Entscheidung einer Weiterbildungsorganisation ein QMS zu implementieren davon beeinflusst wird, wie viele andere im jeweiligen organisationalen Feld bereits ein QMS eigeführt haben. Als Grundlage dafür dienen die oben erwähnten von Reuter et al. (2019) empirisch identifizierten organisationalen Felder. Angenommen wird, dass in diesen organisationalen Feldern jeweils Unterschiede bezüglich der isomorphen Mechanismen zu erkennen sind. Dies bezieht sich insbesondere auf die Effekte von Zwang. Im Sinne ihres Reproduktionskontextes (vgl. Schrader 2010) und ihres Angebotsprofils (das in den organisationalen Feldern berücksichtigt ist) ist davon auszugehen, dass sie unterschiedlich stark auf (gesetzlich fixierten) Zwang reagieren.

Konkret gilt es damit, folgende Hypothesen zu prüfen:

H1 Das organisationale Feld beeinflusst die Entscheidung, ob ein QMS eingeführt wird.

H2 Die Auswirkung gesetzlichen Zwangs auf die Implementation von QMS ist in verschiedenen organisationalen Feldern unterschiedlich.

Bevor diese Hypothesen empirisch getestet werden, werden zunächst das Forschungsdesign und die Methode erläutert. Im Anschluss daran werden die Diffusionsprozesse von QMS beschrieben und diskutiert.

\section{Design und Methodik}

Um die Hypothesen statistisch zu prüfen, wird auf die Daten der wbmonitor-Umfrage 2017 zu dem Themenschwerpunkt „Qualitätsmanagementsysteme“ zurückgegriffen. Die Umfrage wurde gemeinsam vom Bundesinstitut für Berufsbildung (BIBB) und dem Deutschen Institut für Erwachsenenbildung - Leibniz Zentrum für Lebenslanges Lernen (DIE) in Kooperation mit der Justus-Liebig-Universität Gießen als Online-Umfrage durchgeführt. Mit der Ausrichtung auf die gesamte Anbieterlandschaft stellt sie die größte bundesweit regelmäßig durchgeführte Anbieterbefragung dar. $^{6} 2017$ beteiligten sich 1755 Weiterbildungsorganisationen an der Umfrage, was einer Rücklaufquote von $9 \%$ entspricht.

Zur Testung der Hypothesen wird die Methode der Ereignisdatenanalyse genutzt. Für die Verwendung dieses Verfahrens sprechen drei Gründe:

Der erste Grund ist die zur Verfügung stehende Datengrundlage des wbmonitors. In der Umfrage 2017 wurden - neben der aktuellen Nutzung eines QMS - auch die jahresgenauen Zeitpunkte der jeweiligen Implementation und Zertifizierung er-

\footnotetext{
6 Bezüglich der Konzeption der Umfrage sowie der zugrundeliegenden Definitionen von Weiterbildung bzw. Weiterbildungsanbietern sei an dieser Stelle auf das Daten- und Methodenhandbuch des wbmonitors verwiesen (vgl. Koscheck 2010).
} 
hoben. Da der wbmonitor zudem auch Angaben zum jahresgenauen Markteintritt in den Weiterbildungsbereich umfasst, können für einen Zeitraum von 28 Jahren (1990 bis 2017) Verweildauer von und Übergänge zu der Nutzung von QMS nachgezeichnet werden. Im Gegensatz zum aktuellen Status im Umgang mit QMS handelt es sich bei der jeweiligen Verweildauer um ein kontinuierliches Outcome, das wesentlich mehr Informationen enthält und eine adäquate Schätzung von Effektstärken ermöglicht. Sollen Hypothesen also auf der Grundlage der bestmöglichen Daten und aller verfügbaren Informationen getestet werden, legt dies die Anwendung von Ereignisdatenanalysen nahe. Die Daten sind dabei rechts- und linkszensiert: Es treten also (sehr wenige) Organisationen bereits mit einem implementierten QMS in den Berichtszeitraum ein (linkszensiert), und es gibt eine Reihe von Organisationen, die bis zum Ende des Beobachtungzeitraumes kein QMS eingeführt haben (rechtszensiert).

Der zweite Grund schließt an die Aspekte der Aussagekraft und der Belastbarkeit der gewählten Methoden an und zielt auf die Erzeugung anwendungsrelevanter Evidenz (vgl. Schrader 2014). Dies gilt umso mehr, wenn es sich - wie im Fall von Hypothese 2 - um Fragestellungen zur Wirkung konkreter bildungspolitischer Interventionen handelt. Unter diesen Bedingungen stellen sich besondere Anforderungen an die Objektivität und (kausale) Interpretierbarkeit von Befunden. Ereignisdatenanalysen bieten diesbezüglich grundsätzlich Vorteile: Zum einen haben sie Ereignisse zum Gegenstand, die tatsächlich eingetreten oder eben nicht eingetreten sind. Im Gegensatz zu Meinungen, Einstellungen, Haltungen, Einschätzungen oder Interpretationen stellen Ereignisse insoweit objektive Informationen dar, als die Angabe des Datums keine weitere Auslegung erfordert. Obgleich Ereignisdatenanalysen nicht per se kausal interpretierbar sind, bieten sie doch Möglichkeiten, fundamentale Probleme kausalen Schließens zu adressieren: Zunächst können Ereignisse in einer für kausale Interpretationen sinnvollen Reihenfolge untersucht werden. Angenommene Ursachen können also zeitlich vor den theoretisch begründeten und in den Hypothesen formulierten Auswirkungen operationalisiert werden. Ein noch wichtigerer Vorteil der Methode besteht in der Möglichkeit, anstelle von Zuständen die Effekte von Veränderungen im Zeitverlauf auf Veränderungen von interessierenden Outcomes im Zeitverlauf zu schätzen. Dies ist ein zentrales Grundkonzept kontrafaktischer Designs (vgl. Blossfeld und Rohwer 2002). Schließlich können darüber hinaus auch unbeobachtete zeitabhängige Einflüsse bis zu einem gewissen Grad kontrolliert werden.

Der dritte Grund schließt wiederum an der vorhergehenden Darstellung an und verweist auf die besondere Eignung des Verfahrens in Bezug auf die hier ausgeführten theoretischen Annahmen und die daraus abgeleiteten Hypothesen. Letztere richten sich zunächst auf Veränderungen, die sich in den verschiedenen organisationalen Feldern ereignet haben, und die unterschiedlich verlaufen sind. Dieser langwierige Prozess lässt sich nur sinnvoll in seinem historischen Verlauf, nicht aber anhand aktueller Zustände operationalisieren.

Grundsätzlich wollen theoretische Konzepte (in der Soziologie) jedoch ,soziales Handeln deutend verstehen und dadurch in seinem Ablauf und seinen Wirkungen ursächlich erklären“ (Weber 1972, S. 1). Vor dem Hintergrund hoher Komplexität hat sich eine Vielfalt von methodischen Zugängen zur sozialen Wirklichkeit etabliert (vgl. Krüger 2009). Um die theoretischen Annahmen zur Wirkung von Isomor- 
phismen zu beschreiben respektive die daraus abgeleiteten Hypothesen zu testen, erscheint es sinnvoll, sich soweit wie möglich über korrelative Zusammenhänge hinaus an kausal belastbare Aussagen anzunähern. ${ }^{7}$

Um mittels der Ereignisdatenanalyse zu möglichst belastbaren Aussagen im oben beschriebenen Sinn zu gelangen, wurden zwei spezifische Methoden gewählt: Dies ist zum einen ein Piecewise Constant Exponential Model (PCE) und zum anderen ein Semiparametric Transition Rate Model (Cox Model). Beide Modelle zeichnen sich neben den oben beschriebenen grundsätzlichen Vorteilen auch dadurch aus, dass unbeobachtete zeitabhängige Heterogenität kontrolliert werden kann (vgl. Blossfeld et al. 2007). Im PCE wird zu diesem Zweck die Zeitachse in Zeitintervalle aufgeteilt (hier: 7 Intervalle zu je 4 Jahren) und für jedes Intervall eine spezifische Konstante (baseline) in einem einfachen Exponentialmodell geschätzt. Im Cox Model hingegen wird eine unspezifische Baseline-Rate über alle Fälle angenommen und ausschließlich der Effekt der Variablen im Modell geschätzt. Der Nachteil des Cox Models gegenüber dem PCE besteht demzufolge darin, dass kein tatsächliches VorhersageModell konstruiert wird und keine Konstanten geschätzt werden, die einen Einblick in den Verlauf der Baseline-Rate ermöglichen.

Als unabhängige Variablen werden sowohl zeitkonstante als auch zeitabhängige Variablen genutzt. Die zeitabhängigen Variablen werden über jahresgenaue Episodensplits in die Modelle aufgenommen. Aus 1270 Fällen mit gültigen Angaben (listenweiser Ausschluss) werden so 20.390 Beobachtungen. Als zeitkonstante Kontrollvariablen dienen die Siedlungsstruktur der Raumordnungsregion ${ }^{8}$, die Zugehörigkeit zu einem organisationalen Feld, ${ }^{9}$ sowie die Ost-West-Unterscheidung. ${ }^{10}$ Die Siedlungsstruktur kann über Kontrollzwecke hinaus auch als Proxi für die räumliche Nähe von beobachtbaren Weiterbildungsorganisationen des organisationalen Feldes dienen: In städtischen Regionen sollte diese näher, in ländlichen Regionen weiter entfernt sein. Der entsprechende Effekt gibt dann einen Hinweis, welchen Einfluss Distanz auf die Diffusionswahrscheinlichkeit von QMS hat. Die im Modell aufgenommenen zeitabhängigen Variablen dienen der Überprüfung der zentralen Hypothesen. Dabei handelt es sich um die Dichte von Organisationen mit QMS im organisationalen Feld, die gesetzlichen Vorgaben zu QMS in den $W_{B G}{ }^{11}$ und im Zuge der AZWV sowie um die Variable „Zwang“, in der diese gesetzlichen Vorgaben zusammengefasst sind. Die Variable zur Dichte von Organisationen mit QMS im durch die Cluster definierten organisationalen Feld wurde zunächst mit einem Likelihood Ratio Test (LRT) in einem PCE und Cox Model dahingehend geprüft, ob

\footnotetext{
${ }^{7}$ Zum Verständnis von Kausalität bei Ereignisdatenanalysen vgl. Blossfeld und Rohwer (2002). Für eine allgemeine kritische Diskussion von Kausalität in den Sozialwissenschaften vgl. Marini und Singer (1988), spezifisch für die multivariate Statistik vgl. Opp (2010).

8 basierend auf den Daten des Bundesinstituts für Bau-, Stadt- und Raumforschung (BBSR).

${ }^{9}$ Die relative Stabilität der Feldzugehörigkeit wurde geprüft und kann in der Regel angenommen werden (vgl. hierzu Reuter et al. 2019).

10 Diese Information entstammt den wbmonitor-Daten.

${ }^{11}$ Hierzu wurden zum einen diejenigen Länder berücksichtigt, in deren WBGs QMS explizit vorgeschrieben sind (WBG-QMS), und zum anderen diejenigen, in denen Qualitätsstandards definiert wurden (WBGStandards). Dies waren bei QMS: Brandenburg 2012, Niedersachsen 2004, Saarland 2010, Sachsen 2006 und Thüringen 2010. Bei den Qualitätsstandards waren dies: Niedersachsen 1999 und Thüringen 2003.
} 
diese eine grundsätzlich bessere Schätzung des Effekts des organisationalen Feldes auf die Übergangsrate in QMS hat als die Dichte von Organisationen mit QMS insgesamt, also ohne die Definition der Felder.

In den Variablen zu den gesetzlichen Vorgaben werden der Zeitpunkt der Einführung und die Verbindlichkeit der Regelungen für die Organisationen bzw. deren potenzielle, nicht tatsächliche Betroffenheit definiert. Im Fall der Variablen zu Vorgaben zum QMS in den WBG handelt es sich um die Positionierung in einem Bundesland, in dem zu einem Zeitpunkt ein WBG eingeführt wurde, in dem QMS vorgeschrieben oder aber Qualitätsstandards festgelegt sind. Dabei wurde auch berücksichtigt, inwiefern Organisationen von der öffentlichen Förderung und den daran geknüpften Vorgaben zum QMS im WBG ausgenommen sind. Je nach Bundesland betrifft dies in der Regel - neben kommerziellen und wirtschaftsnahen Anbietern - die (Fach-)Hochschulen sowie die beruflichen Schulen, die als primär für Ausbildung zuständige Institutionen einer anderen öffentlichen Finanzierung unterliegen. Bei der gesetzlichen Regelung zur Zertifizierung nach AZWV, deren Vorgaben für das gesamte Bundesgebiet gültig sind, wurde neben dem Zeitpunkt der Einführung ebenfalls die Verbindlichkeit für die Organisation operationalisiert. Diese ist davon abhängig, inwiefern Organisationen im Bereich der SGB III/II geförderten Arbeitsmarktdienstleistungen tätig sind. Letzteres ist bei (Fach-)Hochschulen und beruflichen Schulen gemäß der wbmonitor-Daten nur in Ausnahmen der Fall. Während die Dichte von Organisationen mit QMS im organisationalen Feld zum jeweiligen Zeitpunkt kontinuierlich ist, beschreiben die Variablen zu den gesetzlichen Vorgaben sowie die daraus zusammengesetzte Variable „Zwang“ einen einmaligen Effekt. Alle zeitspezifischen Variablen werden zum jeweiligen Vorjahreswert in den Modellen operationalisiert, um den Anpassungsreaktionen der Organisationen Zeit einzuräumen und Ursache und Wirkung auch zeitlich zu differenzieren.

Die erwähnten berücksichtigten empirisch bestimmten organisationalen Felder (vgl. Reuter et al. 2019) beruhen ebenfalls auf den Daten des wbmonitors und wurden im Anschluss an neo-institutionalistische Annahmen mittels einer Clusteranalyse bestimmt. Theoretisch-konzeptionell wurde dabei an DiMaggio und Powell (1983, 2009) angeschlossen. Trotz der Kritik an dem Konzept, unter anderem zu statisch zu sein (vgl. Walgenbach und Meyer 2008, S. 72 ff.), erweist es sich für die vorliegende Untersuchung jedoch insofern als nützlich, als dass hier nicht der Prozess der Feldstrukturierung ${ }^{12}$ im Fokus steht, sondern retroperspektiv die Diffusion von QMS in die Weiterbildung über einen Untersuchungszeitraum von 28 Jahren nachgezeichnet werden soll. ${ }^{13}$ Hierfür erscheint es sinnvoll, eine Feldkonstruktion zu nutzen, die als relativ zeitstabil angesehen werden kann (vgl. auch Reuter et al. 2019). Der Einschätzung von Hartz folgend, werden u.a. Finanzierungs- und Rechtsbedingungen der Organisationen als feldkonstituierend für die Weiterbildung

\footnotetext{
12 Würde der Prozess der Feldstrukturierung fokussiert, müssten die Beziehungen zwischen Organisationen im Feld genauer analysiert und zur Feldbestimmung genutzt werden (vgl. DiMaggio und Powell 2009, S. 69). Hierzu enthalten die Daten des wbmonitors jedoch keine Informationen - mit Ausnahme von Finanzierungsanteilen, die als Indikator für Kundenbeziehungen angesehen werden können.

13 Dementsprechend wurde im Modell der Ereignisdatenanalyse das Feld als unabhängige und nicht als abhängige Variable aufgenommen.
} 
angesehen, auf deren Basis Weiterbildungsorganisationen ihre Ressourcen wie auch ihre Legitimationen beziehen (vgl. Hartz 2009, S. 136). Dementsprechend wurden neben der Berücksichtigung formaler Organisationsstrukturen (d.h. Art der Einrichtung, Angebotsausrichtung, thematische Breite und Personalstruktur) auch Aspekte der Finanzierung (d.h. Anteil von Weiterbildung am Gesamtumsatz und Zusammensetzung der Einnahmen im Bereich „Weiterbildung“ aus unterschiedlichen Quellen) in die Analyse eingeschlossen, um darüber zentrale Kennzeichen des organisationalen Feldes abzubilden.

Im Ergebnis konstituierten sich vier organisationale Felder der Weiterbildung primär nach den dort anzutreffenden Anbietertypen: Feld 1 enthält die Volkshochschulen, nur wenige Organisationen sind anderer Art. In Feld 2 befinden sich verschiedene Organisationen in vorrangig gemeinnütziger Ausrichtung sowie Staatliche der beruflichen Weiterbildung und Bildungszentren von Kammern. Feld 3 besteht vor allem aus Organisationen in der Trägerschaft einer Kirche, Partei, Gewerkschaft, eines Verbandes oder Vereins, deren Weiterbildungsangebote von einer bestimmten Weltanschauung geleitet sind; in Anlehnung an die Reproduktionskontexte (vgl. Schrader 2010) wird dieses Feld als „Gemeinschaften“ bezeichnet. Im Feld 4 werden schließlich die kommerziellen privaten Weiterbildungsorganisationen gruppiert. Hinsichtlich der Diffusion von QMS ist zu erwähnen, dass in allen Felder ein hoher Abdeckungsgrad durch QM zu erkennen ist. Unterschiede bestehen hinsichtlich der verschiedenen QM-Modelle: Während im Feld 1 der VHS die „Lernerorientierte Qualitätstestierung in der Weiterbildung“ (LQW) das am häufigsten genutzte QMS ist, hat sich in den anderen drei Feldern die jeweils größte Gruppe für ein QM nach der DIN EN ISO 9000-Normenreihe entschieden. ${ }^{14}$

\section{Diffusionsprozesse von QMS in die Weiterbildung}

Im Folgenden werden die Diffusionsprozesse von QMS in die Weiterbildung nachgezeichnet. Dazu werden die Ergebnisse der Ereignisdatenanalyse in einem ersten Schritt dargestellt und in einem zweiten Schritt diskutiert. Von den insgesamt 1755 Weiterbildungsorganisationen, von denen Informationen zum QM vorliegen, können 1270 in der Ereignisdatenanalyse untersucht werden. Da in dieser Stichprobe insbesondere jene Organisationen nicht berücksichtigt werden, die keine Angabe zum Zeitpunkt der Einführung von QMS gemacht haben, bei denen jedoch ein QMS vorliegt, ist der Anteil derjenigen Organisationen, die zum Ende des Beobachtungszeitraums ein QMS aufweisen, im analysierten Sample mit 75,4\% etwas geringer als im gesamten Sample.

Deskriptiv betrachtet zeigen sich zunächst gewisse Unterschiede zwischen den Implementierungsverläufen in den jeweiligen organisationalen Feldern (vgl. Abb. 1). Während kommerzielle private Organisationen des Feldes 4 bis 1999 mit $12 \%$ die höchste Dichte an QMS aufwiesen, wurden sie Anfang der 2000er von den gemeinnützigen/öffentlichen Organisationen (Feld 2) sowie den VHS (Feld 1) überholt.

\footnotetext{
14 Zur ausführlichen Beschreibung des methodischen Vorgehens sowie bezüglich der Merkmale organisa-
} tionaler Felder vgl. Reuter et al. (2019). 


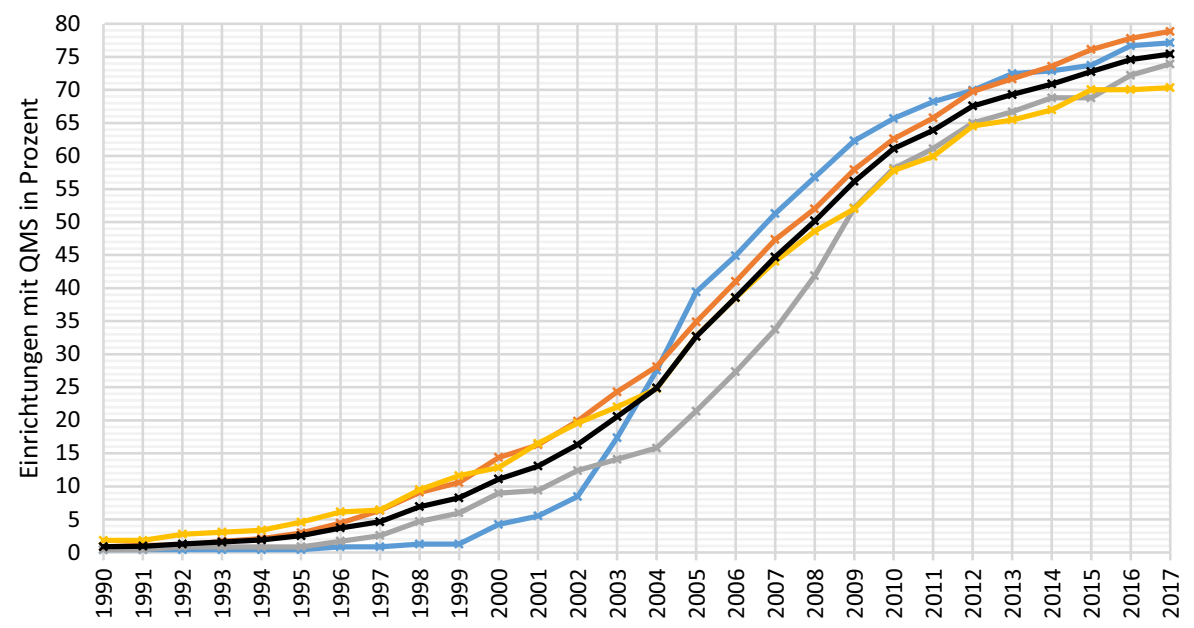

Jahr
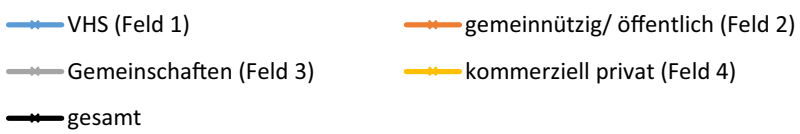

Abb. 1 Implementierungsverläufe von QMS. (Quelle: eigene Berechnung auf Basis der wbmonitor-Umfrage 2017; eigene Darstellung)

2017 bilden die kommerziellen privaten Organisationen schließlich mit $70 \%$ das Feld mit der niedrigsten Dichte an QMS. Organisationen aus dem Feld 4 wenden sich mit ihren Dienstleistungen häufig an private Kunden (vgl. Reuter et al. 2019), deren Finanzierungsentscheidung nicht an das Vorhandensein eines QMS gebunden sein muss. Somit kann von einem geringeren Erfordernis eines QMS ausgegangen werden, sodass das Ergebnis plausibel erscheint. Zum Ende des Beobachtungszeitraums weisen Organisationen im Feld 2 der Gemeinnützigen/Öffentlichen den höchsten Anteil der QMS-Implementationen auf. Auch die Organisationen im Feld 1 der VHS verzeichnen ein höheres Maß an implementierten QMS: Obgleich dieses Feld erst verhältnismäßig spät (ab etwa 2002) begann in größerem Umfang QMS zu etablieren, weist es in 2013 kurzfristig den höchsten Implementierungsgrad auf, bis es 2014 wieder hinter dem Feld 2 der gemeinnützigen/öffentlichen Weiterbildungsorganisationen zurückliegt, welches 2017 mit 79\% die höchste Dichte an QMS aufweist.

Insgesamt zeigt sich jedoch für alle Organisationen, dass sich die Übergangsraten in QMS Ende der 1990er Jahre deutlich erhöht haben, und dass sich in den Jahren ab 2010 ein stagnierender Trend bei nur noch geringen Übergangsraten aber auf hohem Gesamtniveau abzeichnet. Trotz dieses ähnlichen Gesamtverlaufs der vier Felder sind in der Hauptanstiegsphase der Übergänge zu QMS (ab etwa dem Jahr 2000) prägnante Unterschiede zu erkennen. Besonders beachtenswert ist hierbei der Implementierungsverlauf im Feld 1 (VHS), da dieser auffällig steil ansteigt. Das heißt, dass hier ab Beginn der 2000er Jahre in Relation zu den anderen drei Fel- 
dern ein besonders starker Zuwachs an Implementierungen von QMS stattfindet. Vor dem Hintergrund, dass sich dieses Feld durch eine hohe Nutzung des QMS „LQW“ (35\%) auszeichnet, welches im Rahmen des Projekts „Lernerorientierte Qualitätsentwicklung in Weiterbildungsnetzen“" im Zeitraum von 2000 bis 2002 entwickelt wurde (vgl. Ehses und Zech 2002), erscheint dieser Verlauf evident, denn bereits 2001 - also kurz nach der Verabschiedung von LQW 1 - wurden ,fast 120 Anmeldungen zur Qualitätstestierung“" (Zech 2004, S. 8) verzeichnet.

Von zentraler Bedeutung hinsichtlich der in Kap. 3 formulierten Annahmen zu den Einflüssen auf die Entscheidung von Weiterbildungsorganisationen zur Implementierung von QMS und der beiden daraus abgeleiteten Hypothesen sind die Ergebnisse der Ereignisdatenanalyse, worüber Tab. 1 Aufschluss gibt. ${ }^{15}$

Insgesamt ist den Ergebnissen in Tab. $1 \mathrm{zu}$ entnehmen, dass die Übergangsrate in QMS nicht konstant ist, sondern sich im Zeitablauf verändert. Dies verdeutlichen die Konstanten in den PCE-Modellen: Anzunehmen ist, dass hier unbeobachtete zeitspezifische Effekte auf Organisationsebene sowie im gesellschaftlichen Kontext der Organisationen wirken (Periodeneffekte) (vgl. Kap. 4).

Bezogen auf Hypothese 1, dass organisationale Felder die Entscheidung zur Einführung eines QMS beeinflussen, lässt sich festhalten, dass eine Nachahmung beobachtet werden kann. So nimmt die zeitspezifische Dichte von Organisationen mit QMS im organisationalen Feld Einfluss auf die Entscheidung, ein QMS einzuführen. Gemessen wird dies an der zeitspezifischen Dichte von Organisationen mit QMS im organisationalen Feld der jeweiligen Organisation im Vergleich zum Jahr zuvor. Wird der Koeffizient in Modell 1 hierzu umgerechnet ${ }^{16}$, wird deutlich, dass die Übergangswahrscheinlichkeit einer Organisation in ein QMS um ca. 2\% steigt, wenn die Zahl der Organisationen mit QMS im organisationalen Feld um $1 \%$ gegenüber dem Vorjahr gestiegen ist. Unter Kontrolle des Einflusses der sehr wichtigen gesetzlichen Regelungen in Modell 2 ist noch immer, wenn auch leicht schwächer, ein signifikanter Effekt der zeitspezifischen Dichte von Organisationen mit QMS im organisationalen Umfeld sichtbar. Mit jedem zusätzlichen Prozent an Organisationen mit QMS im organisationalen Feld steigt die Übergangsrate im Folgejahr für die entsprechenden Organisationen um ca. 1\%. Diese Ergebnisse werden auch im Cox Model bestätigt. Da dieser Wert auch in Modell 3 stabil bleibt, kann Hypothese 1 bestätigt werden.

Die oben beschriebenen LRTs zeigen jedoch, dass die Dichte von Organisationen mit QMS im definierten Feld zu keiner signifikanten Verbesserung des geschätzten Modells gegenüber der Dichte von Organisationen mit QMS bezogen auf alle Weiterbildungsorganisationen in der gesamten Umwelt führt. Es scheint also, dass QMS - unabhängig der spezifischen organisationalen Felder - ein Querschnittsthe-

\footnotetext{
15 Ursprünglich war geplant, auch die mimetische und normative Isomorphie zu untersuchen, jedoch wurde hierzu leider keine befriedigende Lösung der Operationalisierung gefunden, weshalb diese Formen im Folgenden nicht weiter berücksichtigt werden können. Anders als Hartz (vgl. 2011), welche die Implementierung von LQW evaluativ begleitete, wurde bei dem wbmonitor aufgrund der hohen zeitlichen Differenz zwischen der Implementierung der QMS und der Befragung auf eine Erhebung der Implementierungsgründe verzichtet.

16 Die Berechnungsformel zur Umrechnung der Regressionswerte in Tab. 1 lautet: $\left(\left(e^{\beta_{i}}\right)-1\right) * 100$ (vgl. Blossfeld et al. 2007, S. 100). Im Folgenden werden im Text die umgerechneten Prozentangaben genutzt.
} 
Tab. 1 Ergebnisse der Ereignisdatenanalyse. (Quelle: eigene Berechnung auf Basis der wbmonitor-Umfrage 2017; eigene Darstellung)

\begin{tabular}{|c|c|c|c|c|c|c|}
\hline & \multicolumn{2}{|l|}{ Modell 1} & \multicolumn{2}{|l|}{ Modell 2} & \multicolumn{2}{|l|}{ Modell 3} \\
\hline & PCE & $\mathrm{COX}$ & PCE & $\mathrm{COX}$ & PCE & $\mathrm{COX}$ \\
\hline \multicolumn{7}{|c|}{ Konstante Zeitintervalle mit geschätzter Konstante („,baseline“) } \\
\hline $0-4$ & $\begin{array}{l}-4,09 * * * \\
(0,12)\end{array}$ & - & $\begin{array}{l}-4,13 * * * \\
(0,12)\end{array}$ & - & $\begin{array}{l}-4,38 * * * \\
(0,16)\end{array}$ & - \\
\hline $4-8$ & $\begin{array}{l}-4,22 * * * \\
(0,13)\end{array}$ & - & $\begin{array}{l}-4,26 * * * \\
(0,13)\end{array}$ & - & $\begin{array}{l}-4,50 * * * \\
(0,16)\end{array}$ & - \\
\hline $8-12$ & $\begin{array}{l}-3,93 * * * \\
(0,12)\end{array}$ & - & $\begin{array}{l}-3,96^{* * *} \\
(0,12)\end{array}$ & - & $\begin{array}{l}-4,19 * * * \\
(0,15)\end{array}$ & - \\
\hline $12-16$ & $\begin{array}{l}-3,20 * * * \\
(0,11)\end{array}$ & - & $\begin{array}{l}-3,31 * * * \\
(0,11)\end{array}$ & - & $\begin{array}{l}-3,54 * * * \\
(0,15)\end{array}$ & - \\
\hline $16-20$ & $\begin{array}{l}-3,12 * * * \\
(0,12)\end{array}$ & - & $\begin{array}{l}-3,35^{* * * *} \\
(0,13)\end{array}$ & - & $\begin{array}{l}-3,61 * * * \\
(0,16)\end{array}$ & - \\
\hline $20-24$ & $\begin{array}{l}-3,59 * * * \\
(0,16)\end{array}$ & - & $\begin{array}{l}-3,63 * * * \\
(0,16)\end{array}$ & - & $\begin{array}{l}-3,90 * * * \\
(0,19)\end{array}$ & - \\
\hline $24-28$ & $\begin{array}{l}-4,19 * * * \\
(0,21)\end{array}$ & - & $\begin{array}{l}-4,17 * * * \\
(0,21)\end{array}$ & - & $\begin{array}{l}-4,44 * * * \\
(0,23)\end{array}$ & - \\
\hline $\begin{array}{l}\text { Org. Feld (zeit- } \\
\text { spez. Dichte) }\end{array}$ & $\begin{array}{l}0,016 * * * \\
(0,002)\end{array}$ & $\begin{array}{l}0,015 * * * \\
(0,002)\end{array}$ & $\begin{array}{l}0,006 * * \\
(0,002)\end{array}$ & $\begin{array}{l}0,006^{*} \\
(0,002)\end{array}$ & $\begin{array}{l}0,007 * * \\
(0,002)\end{array}$ & $\begin{array}{l}0,006^{*} \\
(0,002)\end{array}$ \\
\hline $\begin{array}{l}\text { Stadt (Referenz } \\
\text { ländlich) }\end{array}$ & $\begin{array}{l}0,014 \\
(0,066)\end{array}$ & $\begin{array}{l}0,016 \\
(0,066)\end{array}$ & $\begin{array}{l}0,023 \\
(0,067)\end{array}$ & $\begin{array}{l}0,025 \\
(0,067)\end{array}$ & $\begin{array}{l}0,019 \\
(0,066)\end{array}$ & $\begin{array}{l}0,021 \\
(0,066)\end{array}$ \\
\hline AZWV & - & - & $\begin{array}{l}0,727 * * * \\
(0,099)\end{array}$ & $\begin{array}{l}0,700 * * * \\
(0,108)\end{array}$ & - & - \\
\hline WBG-QMS & - & - & $\begin{array}{l}0,291 \\
(0,171)\end{array}$ & $\begin{array}{l}0,290 \\
(0,171)\end{array}$ & - & - \\
\hline WBG-Standards & - & - & $\begin{array}{l}0,763 * * * \\
(0,198)\end{array}$ & $\begin{array}{l}0,731 * * * \\
(0,199)\end{array}$ & - & - \\
\hline Zwang & - & - & - & - & $\begin{array}{l}1,150 * * * \\
(0,181)\end{array}$ & $\begin{array}{l}1,120 * * * \\
(0,187)\end{array}$ \\
\hline \multicolumn{7}{|c|}{ Org. Felder (Referenz VHS) } \\
\hline $\begin{array}{l}\text { Gemeinnützig/ } \\
\text { öffentlich }\end{array}$ & $\begin{array}{l}0,161 \\
(0,092)\end{array}$ & $\begin{array}{l}0,170 \\
(0,093)\end{array}$ & $\begin{array}{l}0,231 * \\
(0,093)\end{array}$ & $\begin{array}{l}0,235^{*} \\
(0,093)\end{array}$ & $\begin{array}{l}0,528 * * * \\
(0,152)\end{array}$ & $\begin{array}{l}0,527 * * * \\
(0,152)\end{array}$ \\
\hline Gemeinschaften & $\begin{array}{l}0,098 \\
(0,107)\end{array}$ & $\begin{array}{l}0,099 \\
(0,107)\end{array}$ & $\begin{array}{l}0,095 \\
(0,107)\end{array}$ & $\begin{array}{l}0,095 \\
(0,107)\end{array}$ & $\begin{array}{l}0,061 \\
(0,185)\end{array}$ & $\begin{array}{l}0,056 \\
(0,185)\end{array}$ \\
\hline $\begin{array}{l}\text { Kommerziell } \\
\text { privat }\end{array}$ & $\begin{array}{l}0,191 \\
(0,103)\end{array}$ & $\begin{array}{l}0,200 \\
(0,103)\end{array}$ & $\begin{array}{l}0,175 \\
(0,104)\end{array}$ & $\begin{array}{l}0,183 \\
(0,104)\end{array}$ & $\begin{array}{l}0,688 * * * \\
(0,167)\end{array}$ & $\begin{array}{l}0,685 * * * \\
(0,168)\end{array}$ \\
\hline \multicolumn{7}{|l|}{ Interaktionseffekte } \\
\hline $\begin{array}{l}\text { Gemeinnützig/ } \\
\text { öffentlich*Zwang }\end{array}$ & - & - & - & - & $\begin{array}{l}-0,497 * * \\
(0,190)\end{array}$ & $\begin{array}{l}-0,490 * * \\
(0,191)\end{array}$ \\
\hline $\begin{array}{l}\text { Gemeinschaften* } \\
\text { Zwang }\end{array}$ & - & - & - & - & $\begin{array}{l}0,061 \\
(0,228)\end{array}$ & $\begin{array}{l}0,071 \\
(0,228)\end{array}$ \\
\hline $\begin{array}{l}\text { Kommerziell } \\
\text { privat* Zwang }\end{array}$ & - & - & - & - & $\begin{array}{l}-0,845^{* * * *} \\
(0,209)\end{array}$ & $\begin{array}{l}-0,828 * * * \\
(0,210)\end{array}$ \\
\hline Ost & $\begin{array}{l}0,329 * * * \\
(0,079)\end{array}$ & $\begin{array}{l}0,335 * * * \\
(0,079)\end{array}$ & $\begin{array}{l}0,288 * * * \\
(0,079)\end{array}$ & $\begin{array}{l}0,293 * * * \\
(0,079)\end{array}$ & $\begin{array}{l}0,313 * * * \\
(0,079)\end{array}$ & $\begin{array}{l}0,316 * * * \\
(0,079)\end{array}$ \\
\hline$N$ & 1270 & 1270 & 1270 & 1270 & 1270 & 1270 \\
\hline Obs & 20390 & 20390 & 20390 & 20390 & 20390 & 20390 \\
\hline
\end{tabular}

$* p<=0,05 ; * * p<=0,01 ; * * * p<=0.001$ 
ma in der Weiterbildung sind: $81 \%, 82 \%, 79 \%$ und $75 \%$ der Organisationen in den jeweiligen Feldern haben ein QMS implementiert.

Organisationale Felder scheinen, wie oben beschrieben, einen spezifischen Orientierungshorizont für die zugehörigen Organisationen darzustellen. Über die zugrundeliegende Definition des organisationalen Feldes im Anschluss an DiMaggio und Powell (vgl. 1983, 2009) hinaus wurde die Bedeutung der räumlichen Nähe zwischen Organisationen ${ }^{17}$ dahingehend getestet, ob sie einen Einfluss auf die Implementierungswahrscheinlichkeit von QMS besitzt, also ob sich Organisationen an anderen Organisationen orientieren, die ihnen räumlich nahe sind. Wie in Tab. 1 dargestellt, haben Organisationen in städtischen Regionen keine höheren Übergangsraten. Geht man davon aus, dass diesen Weiterbildungsorganisationen die entsprechenden Referenzorganisationen räumlich näher sind als dies in ländlichen Regionen der Fall ist, scheint die Nähe keinen signifikanten Einfluss auf die Übergangsrate von QMS zu haben. Dies spricht für eine Bedeutung der kulturellen Dimension des Feldes (vgl. Scott und Meyer 1991). Dieser Befund spiegelt sich in allen drei Modellen wider.

Ebenfalls beachtenswert ist der Effekt für Ostdeutschland. Hier ist die Übergangsrate zu QMS über den gesamten Beobachtungszeitraum im Durchschnitt ca. 38\% höher als in Westdeutschland.

Die Testung von Hypothese 2, dass die Auswirkung gesetzlichen Zwangs auf die Implementation von QMS in verschiedenen organisationalen Feldern unterschiedlich ist, ist Gegenstand von Modell 2 und 3.

In Modell 2 werden zusätzlich die gesetzlichen Vorgaben zu QMS aufgenommen, die im Beobachtungszeitraum Gültigkeit erlangten. Dies sind die AZWV, die WBG der Länder, in denen ein QMS explizit gefordert wird, und die WBG der Länder, in denen Qualitätsstandards festgehalten sind. Auch die gesetzlichen Regelungen zeigen einen deutlichen Effekt: Die Einführung der AZWV im Jahr 2004 hatte eine Erhöhung um $107 \%$ zur Folge, d.h. die Implementierungsrate von QMS hat sich ein Jahr nach Einführung der AZWV nahezu verdoppelt. Auch wenn kein konkretes Fördervolumen für SGB III/II-Maßnahmen, die eine AZWV-Anerkennung voraussetzen, veröffentlicht ist, zeigen die Ergebnisse der wbmonitor-Erhebung aus 2010, dass die AZAV (Nachfolger der AZWV) mit $43 \%$ die am häufigsten verbreitete Anerkennung von Organisationen war und ihr damit eine zentrale Rolle zugeschrieben werden kann (vgl. Ambos et al. 2010, S. 1). Entsprechend plausibel erscheint der starke Einfluss der AZAV auf die Implementierung von QMS. Allerdings ist dieser - wie auch die Effekte der WBG - von einmaliger Art. Der Effekt bei den WBG (soweit darin Qualitätsstandards festgehalten sind) ${ }^{18}$ liegt bei $115 \%$. Hier fällt auf, dass diese zeitlich relativ früh verankert wurden. Zu diesem Zeitpunkt hatten lediglich ca. 10\% (1999) bzw. ca. 20\% (2003) der Organisationen insgesamt ein QMS implementiert. Die explizite Vorgabe von QMS als Förderbedingung in $\mathrm{WBG}^{19}$ hatte hier jedoch keinen signifikanten Effekt. Dies ist insofern plausibel, als dass diese zeitlich sowohl den Qualitätsstandards der WBG als auch der AZWV nachgelagert

\footnotetext{
17 Scott und Meyer erweitern den Feldbegriff von DiMaggio und Powell unter anderem dahingehend, dass lokale und nicht-lokale Beziehungen differenziert werden (vgl. Scott und Meyer 1991, S. 111).

18 Niedersachsen 1999 und Thüringen 2003.

19 Brandenburg 2012, Niedersachsen 2004, Saarland 2010, Sachsen 2006 und Thüringen 2010.
} 
waren, sodass zu diesem Zeitpunkt bereits viele Organisationen ein QMS etabliert hatten, und dies dementsprechend keine zusätzliche Implementierungswelle auslöste. Die Bildungspolitik hat in den Landesgesetzen scheinbar nachträglich fixiert, was bereits gängige Praxis war.

Hauptgegenstand des Modells 3 ist es, Interaktionseffekte zwischen den als Zwang zusammengefassten Variablen der gesetzlichen Vorgaben und den organisationalen Feldern zu testen. Hier zeigen sich ebenfalls deutliche Auswirkungen: Zwang führt demnach bei dem Feld 1 der VHS zu einer Erhöhung der Übergangswahrscheinlichkeit zu QMS von $216 \% .{ }^{20}$ Im Vergleich dazu bewirkt er bei gemeinnützigen/öffentlichen Weiterbildungsorganisationen (Feld 2) eine 39\% geringere Übergangsrate zu QMS als bei den VHS (Feld 1). Bei kommerziellen privaten Organisationen (Feld 4) ist der Effekt auf die Übergangsrate zu QMS sogar um $57 \%$ geringer als bei den VHS (Feld 1). Unter diesem Gesichtspunkt kann auch Hypothese 2 als bestätigt angesehen werden.

\section{Diskussion der Ergebnisse und Ausblick}

Im Folgenden werden in einem ersten Schritt die beschriebenen Diffusionsprozesse von QMS diskutiert - mit Blick darauf, inwiefern die Auswirkungen gesetzlichen Zwangs auf die Implementation von QMS in verschiedenen organisationalen Feldern unterschiedlich sind. In einem zweiten Schritt werden die Befunde hinsichtlich der Annahme der Wechselwirkung zwischen den Isomorphietypen (Zwang, Mimese, Norm) theoretisch rückgebunden und betrachtet. Der Beitrag schließt mit einem kritischen Blick auf die Grenzen der Ereignisdatenanalyse und einem Ausblick.

Erklärungsmöglichkeiten für die Interaktionseffekte werden bei der Ressourcenabhängigkeit und der in der Ereignisdatenanalyse genutzten Operationalisierung gesehen. Bei erster Ansicht wirkt der Befund mit Blick auf die Finanzierungsanteile durch die Arbeitsagenturen/Bundesagentur für Arbeit (AA/BA) irritierend. Im Feld 1 der VHS, das mit 5\% nur einen geringen Finanzierungsanteil von der BA aufweist, ist der Interaktionseffekt mit Zwang viel stärker als im Feld 4 der kommerziellen privaten Organisationen, welche mit $26 \%$ den höchsten Finanzierungsanteil von Arbeitsagentur/Jobcenter haben und damit von der AZWV stärker betroffen sind. ${ }^{21}$ Werden bei zweiter Ansicht diejenigen Organisationen betrachtet, die sich ausschließlich durch Gebühren von Teilnehmenden oder von Betrieben finanzieren, und nimmt man hier ein geringeres Erfordernis eines QMS seitens der Financiers an, fällt auf, dass dieser Anteil bei kommerziellen privaten Organisationen (Feld 4) $28 \%$ beträgt, bei gemeinnützig öffentlichen (Feld 2) $19 \%$ und der Rest unter $10 \%$ liegt, ${ }^{22}$ was die Plausibilität der Ergebnisse unterstreicht.

Eine weitere Erklärungsmöglichkeit wird in der besonderen Bedeutung der Legitimation von öffentlichen Organisationen zu dem Zeitpunkt der AZWV 2004 gesehen.

\footnotetext{
${ }^{20}$ Der in Modell 3 abgebildete Wert zu Zwang bezieht sich auf die Referenzkategorie (VHS).

21 Finanzierungsanteile (vgl. Reuter et al.2019).

22 Quelle: eigene Berechnung auf Basis der wbmonitor-Umfrage 2017.
} 
Durch die Veröffentlichung des Vermittlungsskandals ${ }^{23}$ der BA 2002, der zur späteren Hartz-Reform und in Folge zur AZWV führte, standen besonders öffentlich finanzierte Programme und Organisationen unter Legimitationszwang (vgl. Czommer et al. 2005). Entsprechend plausibel erscheint es, dass gerade diejenigen Felder, die sich in höherem Maße durch Kommune, Land, Bund oder EU finanzieren, in diesem Zeitraum ein QMS implementierten (VHS 39\%, gemeinnützig/öffentlich $25 \%$, Gemeinschaften $28 \%$ und kommerziell privat $10 \%$ ).

Unter der theoretischen Annahme, dass die Isomorphietypen in komplexen Wechselwirkungen zueinander stehen (vgl. Kap. 2), erscheint es einleuchtend, dass im Feld 1 der VHS, das durch eine relativ hohe öffentliche Verantwortung getragen wird, der normative Aspekt (der hier nicht explizit operationalisiert wurde) eine besonders bedeutende Rolle einnimmt und in engem Zusammenhang mit der Gesetzesentwicklung steht. Dies kann statistisch jedoch nicht getestet werden.

Des Weiteren gilt es - im Sinne der kritischen Ergebnisdiskussion - auch die Entstehungszusammenhänge der gesetzlichen Verordnungen in den Blick zu nehmen, um zu prüfen, inwiefern diese als „reiner“ Zwang charakterisiert werden können.

Im Entstehungskontext der AZWV als Verordnung des Bundes lassen sich keine Rückbezüge zu übergreifenden Diskursen erkennen. Vielmehr steht diese in $\mathrm{Zu}$ sammenhang mit Umstrukturierungsmaßnahmen der Arbeitsmarkförderung und damit auch der „Kommission Moderne Dienstleistungen am Arbeitsmarkt 2002“ (vgl. Siefken 2007). Da die Zugänge zu den damit verbundenen Ressourcen gesetzlich geregelt und verordnet sind, lässt sich diese insgesamt als ,Zwang“ charakterisieren.

Komplexer wird es hingegen bei den WBG: Zum Beispiel deutet es in Thüringen darauf hin, dass deren gesetzliche Verordnung in komplexe Diskurse und Praktiken eingebunden ist, wobei zu vermuten ist, dass hier neben Zwang auch normative und oder mimetische Prozesse eine Rolle spielten und diese in Wechselwirkung zu stehen scheinen. So fand in Thüringen seit ca. 1997 eine inhaltliche Diskussion zum Thema „Qualitätssicherung“ im „Forum Landeskuratorium“ statt, dessen Ziel es war, einen trägerübergreifenden Diskurs zu den in den WBG festgelegten Qualitätsmindeststandards zu führen, welcher auf die Veränderung und Erweiterung der Gesetze gerichtet ist. Auch wurde sich aktiv an dem bundesweiten Modellprojekt „Qualitätssicherung in der Weiterbildung“ (DIE) beteiligt, was als Präferenz für eine langfristige und dauerhafte Implementierung von Prozessen kontinuierlicher Qualitätsentwicklung genannt wird (vgl. Kadler 2002, S. 349f.).

Um Wirkungen im Sinne der isomorphen Prozesse zu interpretieren und auch Schlussfolgerungen abzuleiten, ist es damit wichtig, einen kritischen und differenzierten Blick auch auf den Entstehungsprozess von Gesetzen zu werfen, um hier der Gefahr einer pauschalen und gegebenenfalls irrtümlichen Zuschreibung von Ursachen entgegenzuwirken. Dies untermauert einerseits die Annahme, dass (insgesamt) von einer wechselseitigen Beeinflussung unterschiedlicher Akteursgruppen ausge-

\footnotetext{
23 Der sogenannte „Vermittlungsskandal“ der Bundesagentur für Arbeit von 2002 beschreibt den damaligen Vorwurf manipulierter Statistiken bezüglich der Arbeitsvermittlung (vgl. Schütz 2015, S. 175). Der Bundesrechnungshof veröffentlichte im Anschluss an eine Stichprobenprüfung örtlicher BAs eine Pressemeldung (vgl. Presse Info Nr. 13/02 vom 4. Februar 2002). „Dabei wurde eine Reihe von Ungereimtheiten gefunden, die auf eine gezielte Verzerrung der Statistiken in den örtlichen Arbeitsämtern hindeuteten“ (Siefken 2007, S. 185).
} 
gangen werden kann und eine eindeutige Trennung der Isomorphietypen empirisch nur schwer möglich scheint, andererseits trägt der kritische Blick auf Wechselwirkungen jedoch auch zu Schärfung und damit präziseren Identifizierung wie im Fall der AZWV bei.

Mit diesen Ergebnissen wird zugleich auf methodische Grenzen dieser Analyse aufmerksam gemacht. Hierzu zählen die Berücksichtigung nicht aller gesetzlicher Vorgaben, die ein QMS für Organisationen beinhalten. Diese lassen sich beispielsweise in Berlin im Schulgesetz oder bei großen Förderprogrammen wie dem ESF in der Rahmenrichtlinie für Interventionen finden. Hierzu sind jedoch keine Informationen verfügbar, die eine Schätzung deren Wirkung zuließen.

Obwohl Kontrollvariablen in dem Modell aufgenommen wurden, sind neben den genutzten Ereignissen weitere denkbar, die in zeitlichem Zusammenhang mit den operationalisierten gesetzlichen Vorgaben standen und so die Interpretation verzerrt haben könnten.

Darüber hinaus ist zu benennen, dass die Implementierung des QMS lediglich für das aktuell verwendete Modell erfragt wurde. Da ein Modellwechsel durchaus vorstellbar ist, könnte dies zu Verzerrungen der Ergebnisse geführt haben.

Zudem wurde in den Variablen zu den gesetzlichen Vorgaben die potenzielle Betroffenheit definiert, nicht die faktische. Auch dies könnte zu Verzerrungen der Analyse führen, wobei dies in der Konsequenz die Belastbarkeit der Ergebnisse jedoch nicht einschränken, sondern eher stärken würde.

Im Anschluss an diese Befunde ergeben sich weiterführende Forschungsperspektiven: Obwohl die Differenzierung von Mimese und Norm empirisch kaum möglich scheint, da keine umfassende Handlungsnorm besteht respektive ein Orientierungsrahmen im Sinne einer Profession noch nicht so weit fortgeschritten ist (vgl. Hartz 2009, S. 138; Nittel 2000), sollte diese weiter forciert werden, um die Komplexität des Feldes auch empirisch weiter zu durchdringen. Daran anschließend gilt es, die Befunde dahingehend kritisch zu diskutieren, inwiefern differenzierte Umgangsweisen in den verschiedenen Feldern mit der Thematik des QM und mit den vielschichtigen Wirkungen auf unterschiedlichen Ebenen sich zeigen und erklärt werden können. Auch ist der Frage nachzugehen, welche Wirkungen von QMS sich in den jeweiligen Feldern abzeichnen, wobei der Anschluss an den Neo-Institutionalismus und das Konzept der formalen versus aktiven Integration (vgl. Weick 1976, 2009) hierfür geeignet erscheint. Hierbei gilt es, den aktiven und strategischen Umgang von Individuen und Organisationen mit den Ressourcen und Erwartungen ihrer Umwelt, der in neueren Ansätzen des Neo-Institutionalismus stärker konzeptualisiert wird (vgl. Herbrechter und Schemmann 2019; Oliver 1991), zu berücksichtigen.

Open Access Dieser Artikel wird unter der Creative Commons Namensnennung 4.0 International Lizenz (http://creativecommons.org/licenses/by/4.0/deed.de) veröffentlicht, welche die Nutzung, Vervielfältigung, Bearbeitung, Verbreitung und Wiedergabe in jeglichem Medium und Format erlaubt, sofern Sie den/die ursprünglichen Autor(en) und die Quelle ordnungsgemäß nennen, einen Link zur Creative Commons Lizenz beifügen und angeben, ob Änderungen vorgenommen wurden. 


\section{Literatur}

Ambos, I., Koscheck, S., Martin, A., \& Reuter, M. (2018). Qualitätsmanagementsystem in der Weiterbildung. Ergebnisse der wbmonitor Umfrage 2017. Bonn: BIBB. https://www.die-bonn.de/doks/2018qualitaetsmanagement-01.pdf. Zugegriffen: 17. Jan. 2019.

Ambos, I., Weiland, M., Koscheck, S., \& Schade, H.-J. (2010). wbmonitor Umfrage 2010: Wie regelt sich der Weiterbildungsmarkt? Bonn: BIBB. https://wbmonitor.bibb.de/downloads/Ergebnisse_ 20110223.pdf. Zugegriffen: 17. Jan. 2019.

Becker-Ritterspach, J., \& Becker-Ritterspach, F. (2006). Organisationales Feld und Gesellschaftlicher Sektor im Neo-Institutionalismus. In K. Senge \& K.-U. Hellmann (Hrsg.), Einführung in den Neo-Institutionalismus (S. 118-136). Berlin: Springer.

Blossfeld, H.-P., \& Rohwer, G. (2002). Techniques of event history modeling. New approches to causal analysis. London: LEA.

Blossfeld, H.-P., Golsch, K., \& Rohwer, G. (2007). Event history analysis with stata. London: Psychology Press.

Czommer, L., Knuth, M., \& Schweer, O. (2005). ARGE „Moderne Dienstleistungen am Arbeitsmarkt“: Eine Baustelle der Bundesrepublik Deutschland. Arbeitspapier, No. 104. Düsseldorf: Hans-BöcklerStiftung.

Dewe, B. (2005). Der Professionalitätsanspruch der Weiterbildung im Spannungsfeld zwischen Managerialismus, evidenzbasierter Praxis und Teilnehmerverpflichtung. REPORT Zeitschrift für Weiterbildungsforschung, 4, 9-18.

DiMaggio, P. J., \& Powell, W. W. (1983). The iron cage revisited: institutional isomorphism and collective rationality in orginazational fields. American Sociological Review, 48, 147-160.

DiMaggio, P. J., \& Powell, W. W. (2009). Das „,stahlharte Gehäuse“ neu betrachtet: Institutionelle Isomorphie und kollektive Rationalität in organisationalen Feldern. In I. S. Koch \& M. Schemmann (Hrsg.), Neo-Institutionalismus in der Erziehungswissenschaft (S. 57-84). Wiesbaden: Springer.

Egetenmeyer, R., \& Käpplinger, B. (2011). Professionalisation and quality management: struggles, boundaries and bridges between two approaches. European journal for Research on the Education and Learning of Adults, 01, 21-35.

Ehses, C. (2016). Von der Euphorie des Anfangs zu pragmatischen Strategien Bilanz der langjährigen Qualitätsentwicklung der hessischen Volkshochschulen im Kontext von LQW. Hessische Blätter für Volksbildung, 02, 150-160.

Ehses, C., \& Zech, R. (2002). Lernerorientierte Qualitätstestierung in Weiterbildungsnetzwerken. Projekt im Rahmen des Modellversuchsprogramms „Lebenslanges Lernen“ (Abschlussbericht). Hannover.

Forneck, H.-J., \& Wrana, D. (2005). Ein parzelliertes Feld: eine Einführung in die Erwachsenenbildung. Bielefeld: W. Bertelsmann.

Gieseke, W. (1997). Die Qualitätsdiskussion aus erwachsenenpädagogischer Sicht. Was bedeutet Qualität als Erwachsenenpädagoge? In R. Arnold (Hrsg.), Qualitätssicherung in der Erwachsenenbildung (S. 29-47). Opladen: Leske und Budrich.

Hartz, S. (2009). Diffusionsprozesse in der Weiterbildung - eine Analyse aus der Perspektive des Neo-Institutionalismus. In I.S. Koch \& M. Schemmann (Hrsg.), Neo-Institutionalismus in der Erziehungswissenschaft. Grundlegende Texte und empirische Studien (S. 133-159). Wiesbaden: Springer.

Hartz, S. (2011). Qualität in Organisationen der Weiterbildung. Eine Studie zur Akzeptanz und Wirkung von $L Q W$ (1. Aufl.). Wiesbaden: Springer.

Hartz, S. (2018). Qualitätsmanagement und -entwicklung als Gegenstand der Organisationspädagogik. In M. Göhlich, A. Schröer \& S.M. Weber (Hrsg.), Handbuch Organisationspädagogik (S. 567-578). Wiesbaden: Springer.

Hasse, R., \& Krücken, G. (2005). Neo-Institutionalismus (2. Aufl.). Bielefeld: Transcript.

Herbrechter, D., \& Schemmann, M. (2010). Organisationstypen in der Weiterbildung. Eine empirische Analyse aus der Perspektive des Neo-Institutionalismus. In I. K. Dollhausen, T. C. Feld \& W. Seitter (Hrsg.), Erwachsenenpädagogische Organisationsforschung (S. 125-141). Wiesbaden: Springer.

Herbrechter, D., \& Schemmann, M. (2019). Educational Governance und Neo-Institutionalismus in der Weiterbildungsforschung. In I. R. Langer \& T. Brüsemeister (Hrsg.), Handbuch Educational Governance Theorien (S. 181-199). Wiesbaden: Springer.

Kadler, I. (2002). Thüringen. In P. Faulstich \& P. Vespermann (Hrsg.), Weiterbildung in den Bundesländern. Materialien und Analysen zu Situation, Struktur und Perspektiven (S. 334-354). Weinheim: Juventa. 
Käpplinger, B. (2017). Qualitätsentwicklung in der Weiterbildung: Fluch oder Segen? Eine mikropolitische Analyse. In A. Bolder, H. Bremer \& R. Epping (Hrsg.), Bildung für Arbeit unter neuer Steuerung (S. 167-184). Wiesbaden: Springer.

Käpplinger, B., \& Reuter, M. (2017). Qualitätsmanagement in der Weiterbildung. Wiso Diskurs 15, o. S. http://library.fes.de/pdf-files/wiso/13905.pdf. Zugegriffen: 23. Jan. 2019.

Koscheck, S. (2010). wbmonitor 2007-2009. BIBB-FDZ Daten- und Methodenbericht Nr. 4. Bonn: Verlag. https://metadaten.bibb.de/download/642. Zugegriffen: 17. Jan. 2019.

Krücken, G. (2004). Hochschulen im Wettbewerb. In W. Böttcher \& E. Terhart (Hrsg.), Organisationstheorie in pädagogischen Feldern (S. 286-301). Wiesbaden: Springer.

Krüger, H.-H. (2009). Einführung in Theorien und Methoden der Erziehungswissenschaft (5. Aufl.). Opladen: Barbara Budrich.

Luhmann, N., \& Schorr, K. E. (1982). Das Technologiedefizit der Erziehung und die Pädagogik. In N. Luhmann \& K. E. Schorr (Hrsg.), Zwischen Technologie und Selbstreferenz. Fragen an die Pädagogik (S. 11-41). Berlin: Suhrkamp.

Marini, M., \& Singer, B. (1988). Causality in the social sciences. In C. C. Clogg (Hrsg.), Sociological methodology (S. 347-409).

Meisel, K. (2002). Qualitätsentwicklung im Aufbruch. In E. Heinold-Krug \& K. Meisel (Hrsg.), Qualität entwickeln - Weiterbildung gestalten. Handlungsfelder der Qualitätsentwicklung (S. 9-19). Bielefeld: W. Bertelsmann.

Meyer, J., \& Rowan, B. (1977). Institutionalized organisations. Formal structure as myth and ceremony. American Journal of Sociocology, 2, 340-363.

Meyer, J., \& Rowan, B. (2009). Institutionalisierte Organisationen. Formale Struktur als Mythos und Zeremonie. In S. Koch \& M. Schemmann (Hrsg.), Neo-Institutionalismus in der Erziehungswissenschaft. Grundlegende Texte und empirische Studien (S. 28-56). Wiesbaden: Springer.

Nittel, D. (2000). Von der Mission zur Profession? Stand und Perspektiven der Verberuflichung in der Erwachsenenbildung. Bielefeld: W. Bertelsmann.

Oliver, C. (1991). Strategic responses to institutional processes. The Academy of Management Review, 16, 145-179.

Opp, K. D. (2010). Kausalität als Gegenstand der Sozialwissenschaften und der multivariaten Statistik. In C. Wolf \& H. Best (Hrsg.), Handbuch der sozialwissenschaftlichen Datenanalyse (S. 9-38). Wiesbaden: Springer.

Reuter, M., Koscheck, S., \& Martin, A. (2019). Qualitätsmanagement und organisationale Felder in der Weiterbildung. In I. O. Dörner, C. Iller, I. Schüßler, H. von Felden \& S. Lerch (Hrsg.), Erwachsenenbildung und Lernen in Zeiten von Globalisierung, Transformation und Entgrenzung (S. 159-173). Leverkusen: Barbara Budrich.

Schemmann, M. (2018). Institutionentheoretische Grundlagen der Organisationspädagogik. In M. Göhlich, A. Schröer \& S. M. Weber (Hrsg.), Handbuch Organisationspädagogik (S. 187-198). Wiesbaden: Springer.

Schimank, U. (2015). Governance und Professionalisierung. Notizen zu einem Desiderat. In K. Maag Merki, R. Langer \& H. Altrichter (Hrsg.), Educational Governance als Forschungsperspektive. Strategien. Methoden. Ansätze (S. 127-150). Wiesbaden: Springer.

Schrader, J. (2010). Reproduktionskontexte der Weiterbildung. Zeitschrift für Pädagogik, 56, 267-284.

Schrader, J. (2011). Struktur und Wandel der Weiterbildung. Bielefeld: W. Bertelsmann.

Schrader, J. (2014). Analyse und Förderung effektiver Lehr-Lernprozesse unter dem Anspruch evidenzbasierter Bildungsreform. Zeitschrift für Erziehungswissenschaft, 17, 193-223.

Schütz, H. (2015). Delegitimierung und Stabilisierung. Umgang mit Unsicherheit bei der Reform der Bundesagentur für Arbeit. In M- Apelt \& K. Senge (Hrsg.), Organisation und Unsicherheit (S. 175-193). Wiesbaden: Springer.

Scott, R.W., \& Meyer, J.W. (1991). The organization of societal sectors. Propositions and early evidence. In W. W. Powell \& P. J. DiMaggio (Hrsg.), The New Institutionalism in organizational analysis (S. 108-140). Chicago: University of Chicago Press.

Senge, K. (2005). Der Neo-Institutionalismus als Kritik der ökonomistischen Perspektive. (Dissertation). Darmstadt: Technische Universität, Fachbereich Gesellschafts- und Geschichtswissenschaften. http:// elib.tu-darmstadt.de/diss/000620/. Zugegriffen: 23. Jan. 2019.

Siefken, S. T. (2007). Expertenkommissionen im politischen Prozess. Eine Bilanz zur rot-grünen Bundesregierung 1998 - 2005. Wiesbaden: Springer.

Vogel, R. (2007). Ökonomisierung des Öffentlichen? New Public Management in Theorie und Praxis der Verwaltung. In I. S. Jansen, B. Priddat \& N. Stehr (Hrsg.), Die Zukunft des Öffentlichen - Multidiszi- 
plinäre Perspektiven für eine Öffnung der Diskussion über das Öffentliche (S. 152-174). Wiesbaden: Springer.

Walgenbach, P., \& Meyer, R. (2008). Neoinstitutionalistische Organisationstheorie. Stuttgart: Kohlhammer.

Weber, M. (1972). Wirtschaft und Gesellschaft (5. Aufl.). Tübingen: J. C. B. Mohr.

Weick, K. E. (1976). Educational organizations as loosely coupled systems. Administrative Science Quarterly, 21, 1-19.

Weick, K.E. (2009). Bildungsorganisationen als lose gekoppelte Systeme. In S. Koch \& M. Schemmann (Hrsg.), Neo-Institutionalismus in der Erziehungswissenschaft (S. 85-109). Wiesbaden: Springer.

Zech, R. (2004). Vorwort. In R. Zech (Hrsg.), Qualität durch Reflexivität. Lernerorientierte Qualitätsentwicklung in der Praxis (S. 8-10). Hannover: Expressum.

Publisher's Note Springer Nature remains neutral with regard to jurisdictional claims in published maps and institutional affiliations.

Martin Reuter wissenschaftlicher Mitarbeiter an der Professur für Weiterbildung des Instituts für Erziehungswissenschaft an der Justus-Liebig-Universität Gießen.

Dr. Andreas Martin Leitung Nachwuchsgruppe am Deutschen Institut für Erwachsenenbildung - Leibniz-Zentrum für lebenslanges Lernen e. V.

Stefan Koscheck wissenschaftlicher Mitarbeiter und projektverantwortlich für den wbmonitor am Bundesinstitut für Berufsbildung. 